\title{
INVESTIGACION
}

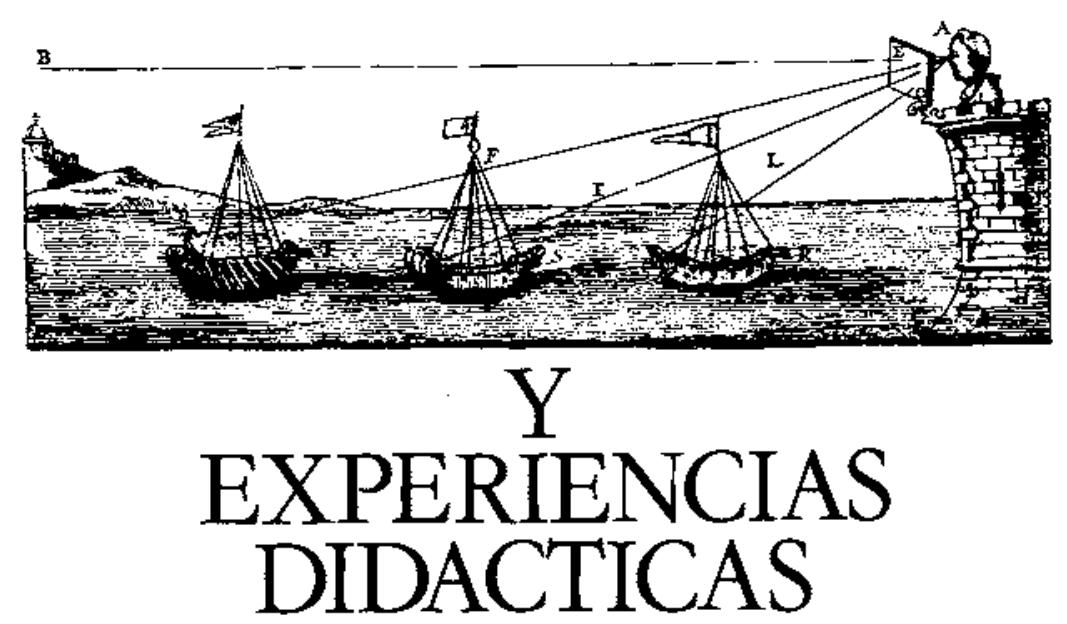

\section{UN ENFOQUE CONSTRUCTIVISTA PARA EL DESARROLLO DEL CURRÍCULO EN CIENCIAS}

\section{DRIVER, $R$.}

Centre for Studies in Science and Mathematics Education. University of Leeds. Leeds (England).

Conferencia invitada en el II Congreso Internacional sobre Investigación en la Didáctica de las Ciencias y las Matemáticas. Valencia 23-25 de septiembre de 1987.

(Versión de J. Martínez Torregrosa).

\section{SUMMARY}

The differences between children's ideas and scientific thinking gives us some indication of the types of changes and the magnitude of those changes which we may be attempting to promote in young people's understanding during school years. In this paper I will review how such changes might be promoted and consider implications for curriculum planning.

\section{INTRODUCCIÓN}

En su introducción al reciente volumen de Nuevas tendencias en la enseñanza de la Fisica, el editor subraya la ausencia de atractivo de la enseñanza tradicional de las ciencias para muchos jóvenes: "Para muchas mentes jóvenes, este enfoque tradicional está tan remoto del mundo en que viven que deciden que la mayor parte del asunto... es bastante irrelevantes (Wenham, 1984, p. 3).

A lo largo de estos últimos años, se ha argumentado reiteradamente la necesidad de ampliar el foco de atención de los cursos escolares de ciencias con objeto de hacerlos así más relevantes para los intereses del individuo y la sociedad moderna. Así, se han desarrollado programas de enseñanza, por ejemplo, que presentan los principios de la ciencia en los contextos que se consideran, en sí mismos, de uso práctico, (p.ej.: óptica de la fotografia, electricidad del hogar, ciencias de la salud).
También se ha argumentado, además, que los cursos deberian reflejar mejor la propia actividad científica; de manera que los estudiantes, como futuros ciudadanos o futuros científícos, lleguen a ser más conscientes de las posibilidades y limitaciones de la ciencia como una empresa humana.

Se trata de tendencias en la concepción del curriculo de ciencias cuya orientación viene determinada por el deseo de suministrar una educación liberal y por la importancia de que los jovenes vean la relevancia de la ciencia para sus propias vidas. En este articulo consideraré una perspectiva adicional que, como intentaré mostrar, también ha de ser tenida en cuenta en nuestra concepción sobre el currículo; una perspectiva que se basa en nuestra comprensión del propio proceso de aprendizaje, en particular de cómo los jóvenes aprenden sobre su mundo físico. 


\subsection{Ideas de los estudiantes sobre fenómenos naturales}

Actualmente existe una amplia literatura, basada en estudios realizados en todo el mundo, que indica que los cstudiantes desarrollan ideas sobre fenómenos naturales mucho antes de que se les enseñe ciencias en la escuela. En algunos casos estas ideas -denominadas de varias maneras: preconcepciones, ideas intuitivas, esquemas conceptuales alternativos, miniteorías, teorías ingenuas - están de acuerdo con lo que va a ensertarsc. En otros casos hay diferencias significativas entre las nociones de los estudiantes y la ciencia que encontrarán en la escuela.

los siguientes ejemplos se han elegido para ilustrar algunas de las clases de ideas que encontramos habitualmente en los razonamientos de los niños en una variedad de fenómenos físicos.

a. Los niños pueden decirnos que un objeto pesará más cuanto mayor sea la altura a la que se eleve, porque cuando cae golpea el suelo más duramente.

b. Ellos argumentan comúnmente que se necesita una «acción» para mantener algo en movimiento. Cuando se empuja un objeto, el «empuje» permanece dentro del objeto y este «empuje» se pierde cuando el objeto se para.

c. Conciben el calor como una clase de «sustancia». Al explicar lo que ocurre cuando se calienta un extremo de una varitla de metal, un alumno de 12 años dijo: "El calor se acumula en una parte hasta que no puede caber más y entonces se mueve a lo largo de la varilla» (Erickson, 1979).

d. "Calor» y «frío" pueden ser considerados como distintos. Hablando sobre lo que ocurre cuando se echa un cubito de hielo al agua, una alumna de 12 años dijo: «Parte del frío dejó el cubo de hielo y pasó al agua» (Erickson, 1979).

e. Las ideas de los niños sobre cómo ven las cosas pueden representarse en términos de "rayos visuales» como muestra el dibujo de la figura 1.

f. La energía es una clase de combustible que se gasta. Discutiendo lo que le ocurre a un juguete de cuerda después de que se ha soltado, una alumna de 13 años reconoce que tiene energía cuando se está moviendo pero que después «se ha gastado».

Niña: Se ha gastado.

Adulto: ¿No puede haberse destruido?

Niña: No.

Adulto: ¿Donde ha ido? "

Niña: El la ha usado. No la ha destruido, la ha usado. (Brook et al., 1986).

g. Las plantas obtienen su comida del suelo.

Se trata solamente de unas pocas ilustraciones de los tipos de ideas que se han identificado en el pensamiento de los jóvenes. Colecciones útiles de artículos sobre figura 1
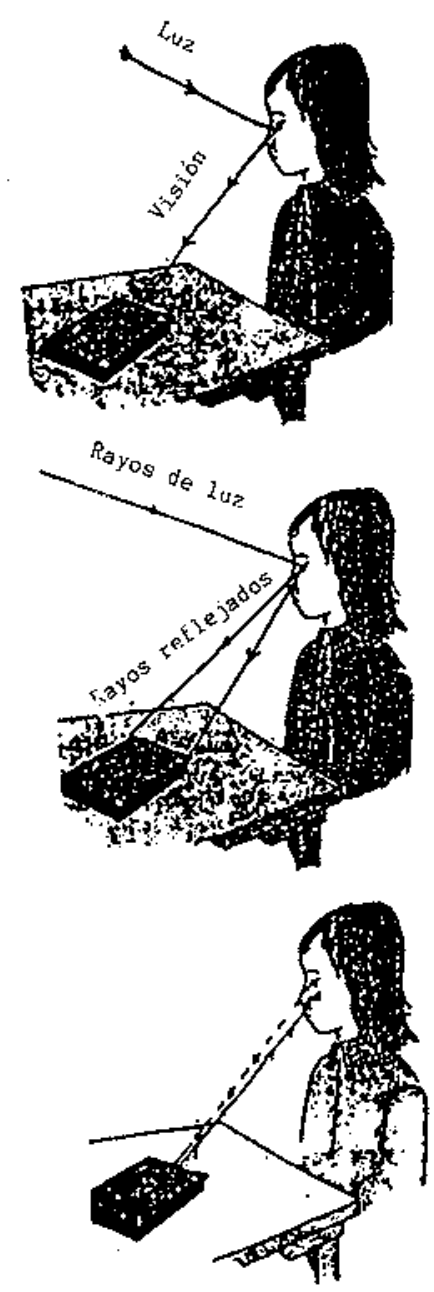

estudios en este campo pueden encontrarse en Helm y Novack (1983), Jung et al (1982), Duit et al (1985), Driver, Guesne y Tiberghien (1985), West y Pines (1985), Osborne y Freiberg (1985).

\subsection{Perspectivas actuales sobre el proceso de aprendizaje}

La investigación sobre las ideas de los estudiantes en ciencias y el modo en que cambian como resultado de la enseñanza puede considerarse como parte de una vi. sión más amplia, hoy emergente, sobre el aprendizaje humano. Una característica clave en las concepciones del aprendizaje, que se utilizan actualmente en psicología cognoscitiva, es la idea de construcciones mentales o «esquemas». Dichos «esquemas» son usados por quienes aprenden, sean bebés o adultos, para interpretar nuevas situaciones. Más aún se admite tambiên que estos «esquemas» son activamente construidos por el que aprende. Quienes aprenden no absorben simplemente lo que se les dice o lo que leen. Desde esta perspectiva, el proceso de aprender implica que quien 
aprende aporta sus «esquemas» o maneras de pensar existentes al enfrentarse a una situación intentando comprenderla. Lo que se aprende no depende, pues, solamente de las caracteristicas de la situación presentada (sea el texto de un libro o un fenómeno físico), sino de los "esquemas» que tiene disponibles el que aprende. En otras palabras, el proceso de aprendizaje es una interacción entre los esquemas mentales del que aprende y características del medio de aprendizaje. Más aún, puesto que construir significado de cualquier nueva situación requiere que el/la que aprende aporte sus «esquemas» sobre la situación y valore su aplicabilidad, en último término, quien aprende es responsable de su propio aprendizaje (Wittrock, 1974).

Esta concepción constructivista del aprendizaje coloca así al que aprende en el centro del proceso de aprendizaje. Lo que se aprende en cualquier programa de trabajo depende de las ideas previas que tienen los estudiantes, las estrategias cognoscitivas de que dispo. nen, y también de sus propios propósitos e intereses. Esta concepción del aprendizaje plantea aspectos fundamentales para la planificación del currículo.

\subsection{Ideas de los alumnos y aprendizaje escolar}

Actualmente admitimos que las ideas previas de los alumnos están presentes en todas las situaciones de aprendizaje en el aula. En las actividades prácticas, las ideas previas de los estudiantes influencian las obser. vaciones que hacen, las inferencias que construyen $\mathrm{e}$ incluso el camino en que estructuran un experimento (Driver, 1983). Lo que aprenden mediante situaciones de aprendizaje más formales, escuchar conferencias, lectura de textos, también está influenciado por las ideas que ya tienen.

Investigaciones realizadas con grandes muestras de alumnos indican que ideas alternativas en áreas tales como mecánica o electricidad pueden persistir a pesar de la ensefianza, y algunas de ellas pueden, incluso, ser utilizadas por estudiantes de física en la Universidad (Viennot, 1979; Sjoberg y Lie, 1981).

Hay una clara evidencia de que las ideas que los niños usan para interpretar fenómenos pueden diferir significativamente de las que se enseñan y que estas diferencias pueden implicar suposiciones sobre el modo en que ocurren las cosas. Dichas diferencias en el modo de ver las cosas pueden actuar como «barreras críticas» (Hawkins, 1978) para la comprensión de ciertos dominios.

Ya hemos hecho referencia a que los estudiantes tienden a pensar que se necesita una "acción" para man" tener algo en movimiento. Esta noción influye en su comprensión de la mecánica clásica pero también in. terfiere en otras áreas. Por ejemplo, cuando se introduce por primera vez la teoría cinética de los gases, la idea de movimiento intrínseco de las partículas es un problema conceptual básico para muchos estudiantes: "¿qué es lo que hace que las partículas se muevan?» y «¿qué es lo que hace que se muevan por primera vez»?» son interrogantes que, con frecuencia, surgen en la discusión (Nussabaum y Novick 1982; Wightman, 1986). Las concepciones de los niños sobre aire, sustancia y peso influyen en cómo entienden cierto número de tópicos (incluyendo las nociones de combustión, ast como los ciclos de materia en los sistemas vivientes).

Es fácil comprender que estos cambios en el pensamiento no tengan lugar en cortos periodos de tiempo. Real. mente, la reestructuración necesaria de ideas puede requerir un periodo de anos y no de una o dos lecciones, y esto puede hacer necesario adoptar una concepción evolutiva en cualquier planificación del curriculo a largo plazo. Como Strauss (1982) sugiere: «una unidad del curriculo que fomenta las actividades y las experiencias de verificación de los niños, si no está basada en una comprensión minuciosa del desarrollo de las competencias de los ninos sobre el concepto, es esencialmente ciega y no deberia esperarse que tuviera exito como instrumento de ensenanza... necesitamos co." nocer el perfil de desarrollo antes de tomar decisiones sobre el contenido curricular, su secuenciación y temporizacion».

Puede extraerse también del trabajo de Strauss un ejemplo útil de la necesidad de comprender la secuencia evolutiva en el pensamiento de los niños. Strauss ha explorado áreas de la experiencia de los niños en las que su rendimiento parece empeorar antes de mejorar (exhibiendo un desarrollo en forma de U). Una de estas áreas que ha sido investigada en profundidad es la evolución de la comprensión de la temperatura por los ni. nos como una cantidad intensiva (Strauss, 1982).

En una de las actividades de la investigación se les presentaba a niños desde 4 a 13 años dos vasijas de agua fría y una tercera vacia (ver figura 2).

\section{figura 2}

Actividad para investigar la comprensión de los estudiantes de la temperatura como cantidad intensiva. (De Strauss, 1982)
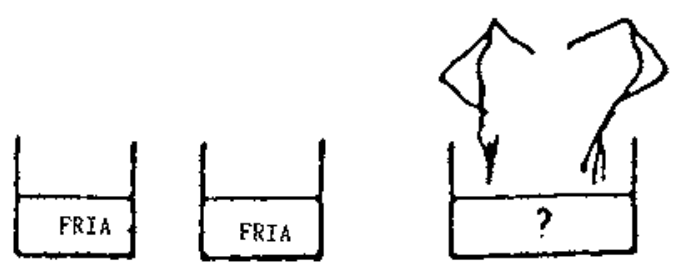

Se les planteaba: «El agua de estas dos tazas está fría. Ahora mira. Las vierto dentro de esta otra taza. ¿Cómo piensas que está el agua ahora? ¿Por qué?» El por- 
centaje de niños que dio la respuesta correcta -que la temperatura de la mezcla seria la misma - se muestra en la figura 3.

\section{figura 3}

Porcentaje de niños que contestan correctamente en la actividad sobre la temperatura.

(De Strauss, 1982)

\begin{tabular}{|cc|}
\hline Edad & $\begin{array}{c}\text { \% de niños que dan } \\
\text { respucstà correcta }\end{array}$ \\
\hline 4 & 65 \\
5 & 35 \\
6 & 15 \\
7 & 50 \\
8 & 30 \\
9 & 55 \\
10 & 55 \\
11 & 85 \\
12 & 85 \\
13 & 80 \\
\hline
\end{tabular}

Strauss identifica tres periodos evolutivos en funcion de las justificaciones que dan los niños. En el primer período los niños más jóvenes dan explicaciones basadas en la identidad, «es la misma agua que había antes» o "Vd. solamente la vertió». El segundo periodo se caracteriza por referencias a la cantidad de agua en la taza. Los niños dicen que la taza con más agua está más fría. En el tercer período los niños vuelven al razonamiento de identidad pero con frecuencia excluyen el volumen como un factor. "La cantidad no importa», «agua a la misma temperatura no cambia su temperatura de repente si la mezclas».

Se trata de un ejemplo particularmente útil para nosotros puesto que indica que, aunque la sofisticación del razonamiento puede aumentar, su rendimiento real, en términos de los factores que tienen en cuenta los niños, puede no mejorar.

Para planificar un curriculo evolutivo puede, también, requerirse una apreciación de las formas en que se relacionan las ideas de los niños. Varios estudios se han realizado en Leeds sobre las ideas de los niños sobre aire y presión del aire (atmosférica), (Engel Clough y Driver, 1985; Miller, Robinson y Driver, 1985). Una de las actividades que hemos usado con niños de 11 , 13 y 15 años les pedía que explicaran qué ocurre cuando un líquido entra en una jeringa. Los tipos de res. puesta dados por diferentes grupos de edad se muestran en la figura 4.

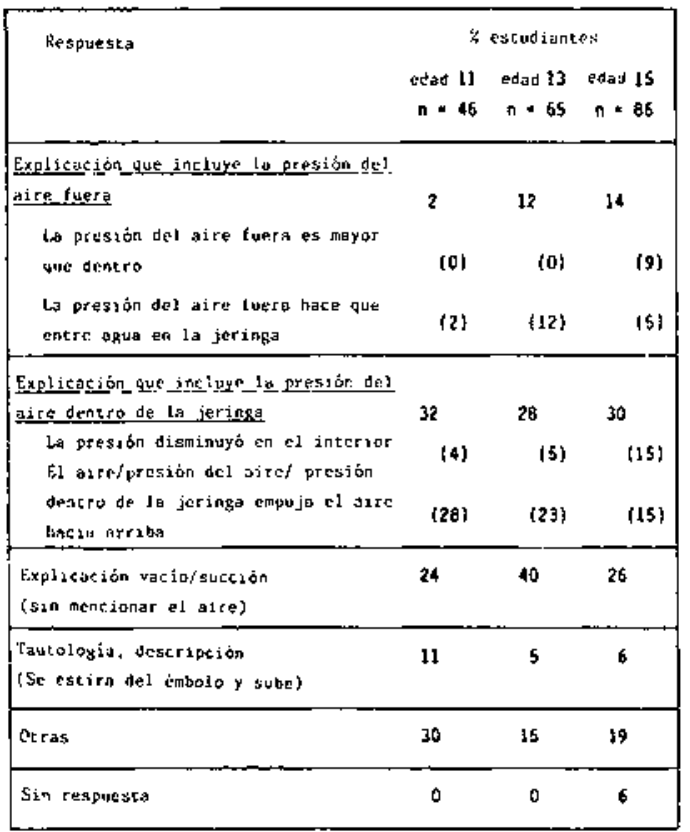


Se advierte un pequeño pero positivo aumento en la proporción de niños que se refieren a la acción del aire en el exterior de la jeringa. Aunque casi todos admiten la existencia de aire, la mayoria se centra solamente en lo que ocurre dentro de la jeringa, explicando la acción en términos del vacío o de la succión como un agente, o del aire o presión del aire en el interior que hace subir el líquido. Esto nos plantea la cuestión de cómo están comprendiendo los niños la acción del aire. A este respecto, una investigación sobre las ideas de los niños relativas al aire y al peso nos da posiblemente algunas ideas. Se les mostró a los niños una balanza de brazos iguales en equilibrio con un recipiente de plástico en cada extremo. Se quito el recipiente de un extremo y se bombeó aire dentro de él. Se les pidió a los niños que predijeran qué ocurriría cuando el recipiente se colocara de nuevo en la balanza. Respuestas en términos de «el aire es ligero y sube» o «el aire no pesan fueron bastante comunes como muestran los resultados de la figura 5 .

figura $S$

Respuestas de estudiantes de secundaria a la actividad de «pesar aire».

\begin{tabular}{|c|c|c|c|}
\hline \multirow[t]{2}{*}{ Respuesta } & \multicolumn{3}{|c|}{ Descudlantets } \\
\hline & $\begin{array}{l}11 \text { sños } \\
11=29\end{array}$ & $\begin{array}{l}13 \text { atios } \\
\pi=56\end{array}$ & $\begin{array}{l}15 \text { años } \\
\pi=6.1\end{array}$ \\
\hline $\begin{array}{l}\text { fl inire es ligero, hasce las } \\
\text { más ligetas o el aire sube }\end{array}$ & 38 & 20 & It \\
\hline El aire no pr'sa & 17 & $1 \mathrm{~s}$ & 8 \\
\hline $\begin{array}{l}\text { El aire liene peso, hace las } \\
\text { cosas más pesadas }\end{array}$ & 28 & 59 & $\$ 3$ \\
\hline Otras & 7 & 2 & 15 \\
\hline So conresta & 10 & 4 & 13 \\
\hline
\end{tabular}

Las dificultades que los nifos tratan de solucionar se ilustran en la siguiente discusión entre niffos de 13 afios sobre una versión de la actividad mencionada utilizando globos en una balanza de brazos iguales.

\section{Daniel: El aire pesa, pesará más ¿verdad?}

Joanne: No.

Daniel: Sí, sí.

Ann: Quedará igual, el aire no pesa nada.

Daniel: Mira, irá hacia abajo - el aire es pesado.

Jaspal: ¡Mirad, escuchad! cuando inflamos el globo, se va para abajo ¿verdad?, porque el aire del globo es más pesado y la gravedad le hace bajar.

Joanne: Sí, pero el aire es ligero, de modo que ¿cómo puede bajar?

Ann: Flota, luego se quedará igual.

Después de hacer la prueba:

Ann: iOh! se va hacia abajo.

Joanne: ¿Pero qué es lo que hace que baje?
Jaspal: Mirad, ino estamos tratando con el aire? Es más pesado que el aire normal de fuera - la gravedad empuja al globo hacia abajo.

Ann: Pero el aire es ligero, hará que el globo flote. Daniel: ¿Cómo se fue hacia abajo, entonces?

Ann: No lo sé. Yo pensaba que se quedaría igual. Joanne: Si fuera ligero iría hacia arriba, ¿verdad?

Jaspal: Mirad, la gravedad lo empuja hacia abajo -ella empuja el aire hacia abajo.

Daniel: Solamente cuando está en el globo.

Hay muchos aspectos de interés en este intercambio, incluyendo la observación de que la evidencia por sí misma no explica el fenómeno. Algunos niños piensan en el aire como algo que "flota a nuestro alrededor» $y$, por tanto, sin peso. Esto tiene como consecuencia que ignoran el efecto del aire sobre las superficies a menos que haya movimiento del mismo (Séré, 1982); para "empujar» algo, se requiere «movimiento» o "peso». $\mathrm{Si}$, por lo que se refiere a los niños, el aire atmosférico no tiene ninguna de estas propiedades, ¿cómo puede ejercer una presión? Si los niños han de construir la noción de la presión atmosférica de modo significativo, entonces tienen que resolver dichas cuestiones.

Los pasos que los niros tienden a seguir en su comprensión científica han sido explorados recientemente en varias áreas entre las que se encuentran la luz (Guesne, 1984, Andersson, 1983), calor y temperatura (Strauss, 1982; Erickson y Tiberghien, 1985), circuitos eléctricos simples (Shipstone, 1985). Los estudios rea* lizados por Piaget y sus colaboradores también sirven como una rica fuente de inspiraciones sobre los caminos de evolución en el pensamiento de los niños. Aun. que seria una simplificación excesiva sugerir que todos los niños siguen la misma ruta en su comprensión de nociones particulares, parece que existen tendencias ge. nerales en el modo en que se construyen ciertas nociones que podrian suministrar información muy útil para el desarrollo del currículo.

Estos estudios se centran sobre el cambio en el razonamiento cualitativo de los estudiantes. Podríamos preguntarnos iqué tiene esto que ver con la capacidad para comprender y utilizar las relaciones matemáticas formales que caracterizan la teoría física? Aquí haré dos puntualizaciones. La primera es para indicar que sabemos que el pensamiento cualitativo juega un importante papel en gran parte de la resolución de proble mas en física. Los estudios que comparan el modo de actuar de expertos y principiantes al resolver problemas de física, muestran que los expertos en primer lugar redescriben el problema de modo cualitativo en términos de conceptos físicos antes de intentar producir una respuesta (Larkin, 1983). Los estudiantes pueden solucionar incorrectamente un problema cuantitativo no porque no puedan manipular las relaciones matemáticas sino a causa de las dificultades conceptuales subyacentes (Gamble, 1986). 
El segundo punto se refiere a nuestra comprensión del desarrollo de las ideas matemáticas de los niños. Se trata de un área de investigación que sería necesario tener en cuenta para elaborar un currículo que respete características evolutivas.

Aunque, como he subrayado antes, se ha hecho alguna investigación básica, en general estamos en un estadio inicial en la comprensión de cómo se construyen los conceptos científicos y, en particular, de cómo el conocimiento físico basado en el formalismo matemático se relaciona con la comprensión intuitiva.

\section{CARACTERISTICAS GENERALES DE LAS IDEAS DE LOS ESTUDIANTES}

\subsection{Introducción}

Como ya se ha indicado, actualmente existen muchos trabajos publicados sobre las ideas iniciales de los estudiantes sobre varios tipos de fenomenos relevantes para la física y otras ciencias. Se han realizado investigaciones en Ias áreas de dinámica, Iuz, electricidad, calor y temperatura, aire y presión del aire, la naturaleza de la materia, y también sobre las ideas cosmológicas de los estudiantes.

Obviamente, sería imposible resumir estos estudios de un modo que les hiciera justicia. En lugar de esto, me centraré en algunas de las características generales de las ideas de los estudiantes que pueden identificarse. En este artículo me referiré a las ideas de los estudiantes porque es el grupo que nos interesa durante la escolaridad. No obstante, ello no quiere decir que los adultos, e incluso los científicos, no piensen de maneras similares cuando se abordan problemas cotidianos.

\subsection{Las ideas de los estudiantes como esquemas activos}

Aunque hay evidencia, a partir de estudios sobre el aprendizaje escolar, de que las ideas de los estudiantes no pueden cambiarse fácilmente mediante la instrucción (Tiberghien, 1986), sería dar una falsa impresión del razonamiento de los niños si se sugiriera que dichas ideas son, en modo alguno, un conjunto fijo o estático de nociones. Podrían calificarse mejor como una serie de posibles "modos-de-ver» de que disponen los estudiantes y que pueden ser ensayadas en situaciones novedosas. Como Claxton (1986) sugiere, son herramientas para aprender y forman una base para adquirir nuevas comprensiones mediante una forma de razonamiento analógico. Las experiencias tempranas de los ninos pequerios empujando objetos en el suelo - una acción que produce movimiento - es usado como esquema básico al cual se asimilan otros tipos de movimientos forzados; el juego con el agua puede suministrar un esquema básico para desarrollos posteriores de la noción de flujo.
Los niños tratan de entender situaciones nuevas «viéndolas como" algo que conocen o reconocen. Al explorar las vigas de una balanza de un solo brazo, hasta entonces desconocida para él, un niño puede, en breve tiempo, decir «es como las balanzas». La nueva situación ha sido asimilada dentro de la noción de balanza del nifo, donde el comportamiento de las balanzas se «da por supuesto» en vez de algo que debe explicarse.

Esta imagen dinámica del razonamiento del niño se pone claramente en evidencia cuando situaciones novedosas se asimilan dentro de esquemas inapropiados. Por ejemplo, incluso estudiantes universitarios de ffsica cuando se les presentan dos masas iguales colgadas a diferentes alturas en una polea predecirán que las dos masas se moverán hasta que se queden al mismo nivel (Gunstone y White, 1981) usando argumentos como use nivelarán como una balanza». Los niños se sorprenden frecuentemente de que la presión en un líquido actúe en todas direcciones. Tienden a usar su esquema para el peso de un cuerpo sólido, una fuerza que actúa hacia abajo solamente, para interpretar lo que ocurre en un líquido (Engel Clough y Driver, 1985).

\subsection{Las ideas de los estudiantes son coherentes dentro de su modo de pensar}

Una característica importante para la comprensión del pensamiento de los niños es apreciar que las concepciones que usan pueden ser coherentes vistas desde la perspectiva del nifto. En dinámica, por ejemplo, sabemos que los estudiantes normalmente asocian fuerza constante con movimiento constante una noción bien adaptada al mundo con rozamiento. Lo que quieren decir con «fuerza» no obstante no es to mismo que lo que entiende como tal un físico. En casos como éste, vemos que las concepciones de los estudiantes tienen sentido dentro de su propia manera de ver las cosas. En este sentido, no están equivocados, simplemente interpretan de un modo diferente. Para comprender el pensamiento de los estudiantes, por tanto, es importante preguntarse ide qué manera tiene sentido esta idea? En un estudio reciente sobre las nociones de los niños sobre el proceso de disolución, algunos niños más pequeños dijeron que el azúcar cuando se agitaba en el agua se convertía en agua. Pruebas posteriores indicaron que para ellos agua significa cualquier líquido transparente y, en estos términos, su respuesta -que el azúcar estaba todavía allí aunque se habja convertido en agua - tiene sentido.

\subsection{EI razonamiento está ligado a un contexto especiffico}

Es un rasgo característico de las ideas de los niños que son limitadas en su generalidad y tienden a estar relacionadas con contextos especificos. Situaciones que pueden ser "vistas" como similares desde un punto de vista cientifico pueden ser interpretadas por los nintos utilizando nociones diferentes. Por ejemplo, la mayor 
parte de un grupo de estudiantes de secundaria, explicó lo que sentían cuando tocaban una cuchara caliente en términos de calor o energia calorifica que abandonaba su mano. En lugar de ello, muchas de las respuestas asignaban la propiedad de "frialdad» al objeto (Engel, Clough y Driver, 1985).

Inchuso en algunos casos donde se nota la inconsistencia, puede no ser vista como un problema. Esto señala una diferencia significativa entre pensamiento cotidiano y pensamiento científico donde la coherencia y parsimonia son criterios importantes. En el pensamiento cotidiano lo que importa es que nuestras ideas sobre una situación «se ajusten» a esa situación permitiéndonos, si es necesario, predecir y actuar dentro de ella: las ideas pueden estar ceffidas al contexto y ser de generalidad limitada y permitirnos, sin embargo, hacer esto. Así, el pensamiento cotidiano puede consistir en una colección de ideas específicas para cada situación cuyas interrelaciones no son de importancia crucial.

Otra característica más que advertimos sobre las ideas de los niños es que un individuo puede responder a la misma situación usando diferentes ideas en diferentes ocasiones. Esto indica que el individuo tiene disponible varios "modos de ver» una situación. Es probable, incluso, que sea este pluralismo el que haga posible que pueda ocurrir el aprendizaje y el cambio conceptual.

\subsection{Diferenciación de ideas}

Las ideas que usan los niños pueden reflejar nociones relativamente no diferenciadas. Por ejemplo, las ideas de los niños sobre el peso incorporan con frecuencia aspectos de volumen, solidez y presión. La noción que tienen los niños sobre el "aire» puede tener connotaciones de un medio general para la transmisión de efectos; por ejemplo, a menlido asocian la presión del aire con la gravedad. La electricidad es una noción no bien definida que incorpora potencia, corriente y energía. Este caracter indiferenciado de algunas nociones permite que los niños pasen de un significado o aspecto a otro sin ser necesariamente conscientes de ello (un factor que explica de alguna manera las inconsistencias en su pensamiento).

\subsection{Del pensamiento perceptivo al conceptual}

Las ideas de los niños se desarrollan de varias maneras cuando se van haciendo mayores. En particular, en los niños más jóvenes advertimos que sus nociones tienden a estar dominadas por las percepciones; cuando una sustancia se quema la materia desaparece, cuando bebes con una pajita el líquido sube a la boca a causa de la "succión». En ninguno de estos casos se plantean la existencia de entidades tales como el aire o los gases, que no pueden ver o sentir.
En cambio, cuando los niños son mayores, incorporan más ideas que se refieren a entidades que no se perciben directamente.

\subsection{Atención a las propiedades más que a las interacciones}

El pensamiento de los nif̂́os pequeños se caracteriza también por una tendencia a dar interpretaciones en términos de propiedades de los objetos antes que en términos de interacciones entre sistemas. Por ejemplo, las diferentes sensaciones que se producen al tocar un metal o un plástico son explicadas considerando que el metal es una sustancia intrínsecamente fría y el plástico cálida. El peso, una noción extremadamente compleja para los pequeños, se ve como una propiedad de un cuerpo más que como una interacción entre un objeto y la Tierra.

\subsection{Razonamiento causal}

Estudios realizados en varias áreas seffalan el hecho de que el razonamiento de los niños se centra sobre estados cambiantes más que en estados de equilibrio. Advertimos, por ejemplo, que los niños reconocen que actúa una fuerza cuando se observa movimiento, pero es menos probable que consideren la existencia de fuerzas en sistemas en equilibrio estático (concibiendo éste como el «estado natural» del sistema».

La idea de que es el cambio lo que requiere explicación está en la raíz del razonamiento causal de los ninos. Al dar explicaciones, el razonamiento de los niños tiende a seguir una secuencia causal lineal. Ellos postulan una causa que produce una cadena de efectos en orden lineal temporal. Este tipo de razonamiento se ha identificado en las ideas de los ninos sobre varios fenomenos y se ha sugerido que tiene sus origenes en esquemas de acción tempranos de los niños pequeños (Anderson, 1985). Una caracterfstica de este tipo de razonamiento es la tendencia a pensar en direcciones preferentes más que percibiendo la simetría en las interacciones entre sistemas. Por ejemplo, al considerar una olla de agua sobre un mechero, los niños tienden a pensar en términos direccionales con el mechero como fuente, suministrando calor a un receptor.

Una consecuencia de esta manera direccional de pensar, que difiere de forma fundamental de la explicación en el pensamiento científico, es que los procesos que un cientifico considera de una manera reversible, no son necesariamente vistos de esta manera por los ninos. Por ejemplo, ellos entienden que una entrada de energía puede cambiar el estado de una sustancia de sólido a líquido (la energfa urompe los enlaces») pero tienen dificultades para comprender el proceso inverso cuando un líquido solidifica. (iEllos interpretan la energía como una «acción sobre el sistema» para romper los enlaces y se quedan con el problema de explícar de dónde vuelven los enlaces!). 


\section{HACIA UNA CONCEPCIÓN CONSTRUC- TIVISTA DEL DISEÑO DEL CURRÍCULO}

\subsection{Introducción}

Las diferencias entre las ideas de los niños y el pensamiento científico nos da alguna indicación de los tipos de cambios y la magnitud de los mismos que podemos estar intentando fomentar en la comprensión de los jóvenes durante los años escolares. En esta sección revisaré cómo podrían promoverse dichos cambios y consideraré implicaciones para la planificación del currículo.

\subsection{EI aprendizaje como cambio conceptual}

Cómo fomentar el cambio conceptual es actualmente un asunto de interés y objeto de investigación. Examinemos brevemente algunas opciones.

La primera opcion, practicada ampliamente, es ignorar las ideas previas de los estudiantes y basar la enseñanza únicamente en la propia estructura de la materia. La idea es que si la estructura de un tópico, sea óptica, dinámica, fotosintesis, se presenta de un modo bien organizado en términos de relaciones formales entre los conceptos científicos, esto permitirá que los alumnos desarrollen esta estructura conceptual por sí mismos. Conocemos ahora los problemas inherentes a dicho enfoque. Los investigadores advierten, por cjemplo, que las ideas previas pueden persistir a to largo del nivel universitario a pesar de dicha instrucción (Viennot, 1979; Reif, 1986). Este enfoque también conduce a una compartimentación del pensamiento de los estudiantes, el conocimiento escolar está separado del conocimiento cotidiano y solamente se usa para contestar problemas «escolares» o preguntas de examen (Solomon, 1983). Puede haber también un grupo de estudiantes para quienes las ideas enseriadas son tan diferentes de las propias que no ven ningún valor a tomarse el estudio en serio. La ciencia para ellos es sólo un conjunto de reglas y relaciones sin significado que no parecen tener ningún contacto con su experiencia y eligen no participar en el aprendizaje.

Una alternativa a la "transmisión verbal», en sus distintas formas - conferencias, libros de textos o demostraciones-, es dar oportunidades a los estudiantes para «descubrir por cllos mismos», es decir, basar el aprendizaje de los estudiantes en la experiencia empírica. Desafortunadamente dichos «métodos de des" cubrimiento" han fracasado; no porque los estudian. tes sean incapaces de investigar por si mismos y extraer inferencias de sus observaciones, sí que son capaces de hacerlo, sino porque no «descubren" necesariamente lo que se pretendia (Atkinson y Delamont, 1976; Koertge, 1970; Wellington, 1981). La experiencia por si misma no basta. Incluso, en muchos casos la evidencia empírica se usa por los estudiantes para reforzar sus nociones previas más que para estimular el cambio (Gunstone y White, 1981, Rowell y Dawson, 1983).
Actualmente se está explorando un tercer enfoque del problema. Este supone que las ideas previas de los alumnos son un punto de partida necesario y que se debería diseñar la instrucción para permitir que estas ideas se desarrollen y cambien. El proceso de cambio conceptual ha sido analizado desde varias perspectivas teóricas (West y Pines, 1985) y se han publicado va. rios estudios para investigar los modos de fomentar el cambio conceptual en las clases de ciencias (Nussbaum y Novick, 1982; Hewson y Hewson, 1984; Champage et al., 1982; Osborne y Freyberg, 1985). Es característico de estos planteamientos la inclusión de oportunidades para que los estudiantes hagan explícitas sus ideas, oportunidades para la clarificación e intercambio de ideas, experiencias que ponen en cuestion las no. ciones que tienen los estudiantes (situaciones de conflicto), oportunidades para la introducción de nuevas ideas o para la reestructuración de ideas por el profesor y oportunidades para utilizar los conceptos en situaciones variadas. Quizás el rasgo más característico de estos abordes es el énfasis que se pone en que los estudiantes sean conscientes de su propio aprendizaje y amplien su razonamiento cotidiano para que pueda ser usado con confianza en un rango más amplio de situaciones.

\subsection{Una perspectiva constructivista de currículo}

Esta perspectiva se basa en una concepción de quien aprende como parte activa e interesada en el proceso de aprendizaje e implicado en aportar sus conocimientos previos para construir significados en situaciones nuevas. No se concibe el conocimiento científico como "objetivo» sino como construcción social, un producto del esfuerzo colectivo de la Humanidad. Si el objetivo de la ciencia escolar es que los niños entiendan la concepción del mundo de los científicos no se conseguirá esto mediante enfoques ingenuos de «naturaleza interrogativa». La socialización es una parte im. portante del proceso.

En este proceso, el curriculo - más que concebirse como «lo que debe ser aprendido»-, se concibe como el conjunto de experiencias mediante las cuales los que aprenden construyen una concepción del mundo más cercana a la concepción de los científicos. El que aprende ilega a clase con ideas previas que neccsitan ser tenidas en cuenta puesto que influyen en los significa. dos que se construyen en las situaciones de aprendiza. je. Existen objetivos de aprendizaje - -alguna versión de las ideas y modos de pensar de los científicos. También existen limitaciones que provienen del ambiente de aprendizaje en las escuelas, disponibilidad de tiempo, recursos materiales, al igual que otras limitaciones más sutiles debidas a las expectativas del profesor y estudiante sobre el conocimiento, la ciencia, las escuclas y las aulas y sus papeles en ellas. Durante una secuencia de aprendizaje los que aprenden deben recorrer un camino desde su estado de conocimiento presente a cierto estado de conocimiento futuro. La cuestión curri- 
cular es ¿cuáles son las actividades de aprendizaje que hacen posible que esto ocurra efectivamente?

Esta cuestión puede ser contestada en parte analizando el propio dominio de conocimientos. No obstante, puesto que lo que se aprende de una situación depende de la situación y de lo que aporta quien aprende en términos de propósitos y esquemas, el diseño y selección de experiencias de aprendizaje debe implicar, en último término, investigaciones empíricas de aprendizaje en el aula. Este proceso reflexivo se ilustra en la figura 6 .

\section{figura 6}

Un modelo constructivista para el desarrolto del curriculo.

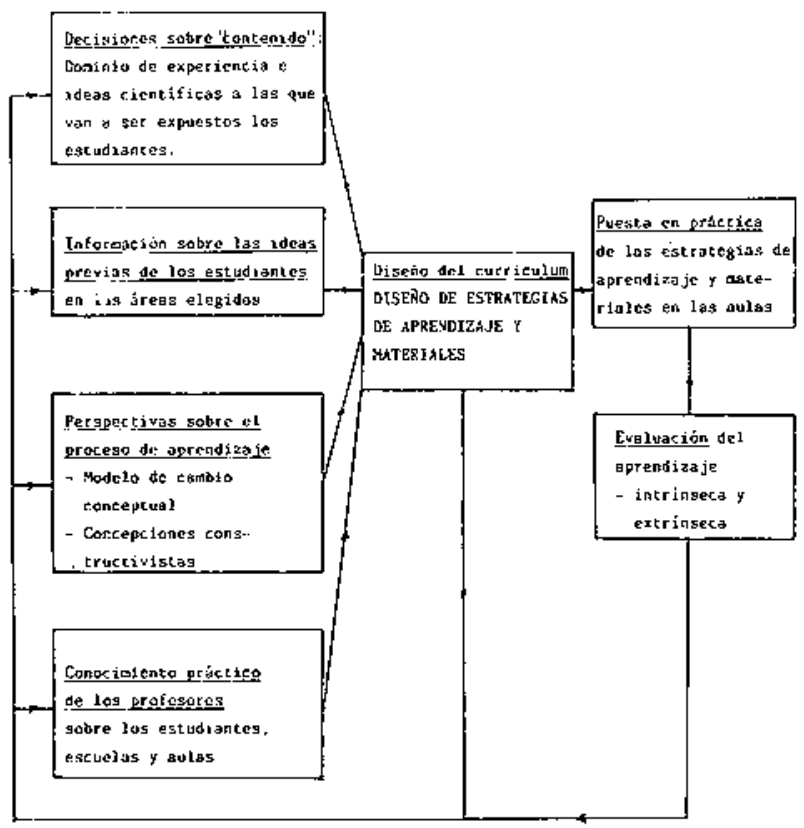

El papel del profesor se hace, así, mucho más complejo que el de un director de un «sistema de transmisión». Como mediador entre el conocimiento de los científicos y las comprensiones de los niños, se requiere del profesor/a que actúe como diagnosticador del pensamiento de los niños y al mismo tiempo que lleve en su cabeza un mapa del dominio conceptual que permita sugerir actividades apropiadas y negociar significados.

\subsection{Diseñando programas de aprendizaje}

¿Cuáles son las exigencias para el desarrollo de materiales y programas de aprendizaje basados en esta perspectiva? Un proyecto de investigación y desarrollo (EI "Children's Learning in Science Project» en Leeds) en el cual he estado implicada durante los últimos años ha explorado esta cuestión (Driver y Oldham, 1986). Como consecuencia del trabajo en colaboración de un equipo de profesores e investigadores se han desarro- llado algunos esquemas experimentales (CLIS, 1987). Existen algunas características que subyacen en el diseño de estos esquemas:

3.4.a Una secuencia de enseñanza basada en el cambio conceptual

La enseñanza está estructurada en torno a una secuencia de actividades que está diseñada para partir de las ideas de los estudiantes y fomentar el cambio en su pensamiento. La secuencia se muestra en términos generales en la figura 7 .

\section{figura 7}

Estructura general de la secuencia de enseñanza.

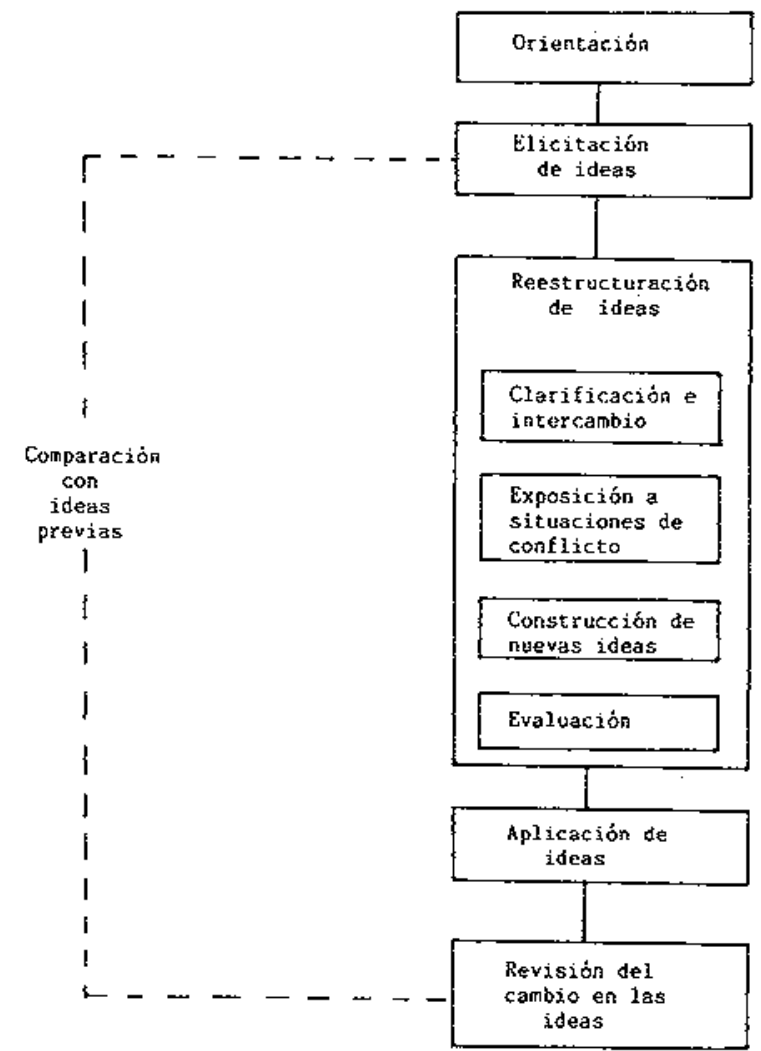

Después de una sesión inicial de orientación destinada a despertar la atención de los estudiantes y su interés por el tema, se destina algún tiempo a que los alumnos revisen y discutan sus propias ideas o modelos. Esta fase de explicitación se inicia generalmente en pequeños grupos. Se pide a cada grupo que represente sus ideas en un póster o por otros medios y que las presenten a toda la clase. Se identifican semejanzas y diferencias en las ideas iniciales de los estudiantes y se sefralan aspectos para posterior consideración. Los posters permanecen expuestos como un registro durante el resto de la unidad del trabajo y más tarde pueden ser corregidos o comentados. 
La fase de reestructuracion ha supuesto el uso de un amplio rango de estrategias. Dichas estrategias pueden incluir:

- Confrontar las ideas de los estudiantes con contraejemplos. Esto puede promover insatisfacción con las concepciones iniciales, pero por sí mismo no genera concepciones alternativas.

- Ampliar el rango de aplicación de una concepción. Las ideas de los estudiantes pueden ser un recurso que puede ser extendido.

- Diferenciación de una concepción. En muchas áreas las concepciones de los estudiantes son globales y vagas; experiencias particulares pueden ayudarles a clarificar y diferenciar sus nociones (por ejemplo: fuerza y energía, calor y temperatura).

$\rightarrow$ Levantar puentes de experiencias para una nueva concepcion.

- La construcción de una concepción alternativa. En algunos casos las ideas previas de los estudiantes no guardan relación con la concepción científica y puede ser necesario construir un modelo alternativo.

- Desarrollar la base para una nueva teoría usando un modelo o analogía.

La secuenciación de la clase ofrece, en este momento, oportunidades para que los estudiantes prueben y apliquen sus concepciones revisadas de varias maneras. Esto puede suponer actividades de construcción práctica, escritura libre o solucionar problemas de libro más convencionales.

Al final de la secuenciación del tema se les da a las clases la oportunidad de revisar la extensión y maneras en que ha cambiado su pensamiento. Sus ideas iniciales pueden estar modificadas o pueden haberse construido nuevas y compararse con las iniciales.

\section{4.b. El contexto de las actividades de aprendizaje}

Las actividades de aprendizaje se han elegido, en la medida de lo posible, para que llamen la atencion (sorprendentes, divertidas) o para ser planteadas en contextos que tenian sentido para los estudiantes (por ejemplo: parte del trabajo sobre energía se ha planteado en contextos domésticos y ha supuesto lectura de contadores, el uso de recibos de combustibles). Sabemos que el conocimiento está embebido en un contexto. Si se han de desarrollar esquemas, el contexto en el cual se haga esto puede ser importante para mantener la atención y facilitar más tarde la aplicabilidad de las concepciones.

\section{4.c. Un ambiente de aprendizaje no amenazante}

Un ambiente de aprendizaje que requiere que los estudiantes expliciten sus ideas y que prueben nuevos caminos de pensamiento podría ser muy amenazante. Si los esfuerzos de los estudiantes son evaluados dema- siado pronto por el profesor o por otros estudiantes, tenderán no a experimentar sino que querrán que se les explique, cortocircuitando, por tanto, el proceso de construcción de conocimientos.

Se ha intentado crear este ambiente no amenazante en las clases desarrollando estrategias que permitan que los estudiantes expresen sus ideas en un modo organizado mediante trabajo en grupo, exposiciones de posters, etc. También se requiere en muchos casos que los profesores cambien sus hábitos de dirección de la discusión en la clase de modo radical, evitando preguntas cerradas, aceptando distintas sugerencias de la clase sin exigir una conclusión prematura sobre un punto.

\section{4.d. Trabajo en pequeño grupo}

La importancia de la discusión para permitir que quie. nes aprenden expresen sus ideas a ellos mismos ha sido reconocida desde hace antos. Pequeños grupos de alrededor de cuatro estudiantes forman la unidad estructural en torno a la cual tiene lugar el esquema de actividades. Las actividades de grupos suponen discutir y expresar teorías o ideas sobre un tópico, diseñar experimentos para comprobar sus ideas, diseñar mo. delos más complejos para presentar experiencias, aco. meter actividades de construcción práctica en las oue se apliquen las concepciones.

\section{4.e. Metacognición}

Se utiliza aqui este término para discutir el proceso por el cual los estudiantes reflexionan sobre su propio conocimiento y sobre cómo está cambiando. Los estudiantes suelen creer que aprender ciencia es «ingerir» hechos aislados. Las estrategias que favorecen que los estudiantes reflexionen sobre su propio aprendizaje les ayudan a apreciar que está en juego un proceso de cam. bio conceptual y también que su conocimiento es estructurado e interrelacionado. Las técnicas que hemos usado para fomentar este proceso incluyen que los estudiantes comparen sus ideas al principio y al final de una secuencia de aprendizaje y también que escriban anotaciones personales sobre el aprendizaje (pequeños cuadernos en los que ellos registran sus reacciones ante los temas, notas sobre lo que ellos piensan, cosas que encuentran difíciles, etc.).

Estos esquemas se han utilizado con clases, orientán. dose el proceso de aprendizaje como se ha descrito más arriba. Fundamentando el aprendizaje de esta forma, hemos podido averiguar en qué medida los estudiantes han sido capaces de construir y usar las ideas deseadas. También hemos podido identificar algunas de las dificultades conceptuales encontradas y advertir los caminos comunes que los estudiantes siguen en su comprensión.

Esta retroalimentación obtenida del aprendizaje en el 
aula se ha utilizado para hacer posteriores modificaciones en los esquemas, alli donde era necesario. También se ha usado para preparar materiales para dar a otros profesores de ciencias algunas ideas sobre las concepciones previas que los estudiantes pueden tener sobre un cierto tópico, los caminos que pueden seguir en su pensamiento y los obstáculos conceptuales que pueden encontrar.

\section{CONCLUSIÓN}

La primera sección del artículo ha intentado ilustrar como podría realizarse un currículo evolutivo. La cuestión clave en esta argumentación es qué entender por curriculo. Si se concibe como un cuerpo de conocimientos que debe ser transmitido al que aprende, su desarrollo puede ser visto como aproblemático. Simplemente decidimos qué es lo que la gente necesita saber. EI problema, como hemos visto, es que pueden haber discordancias entre nuestros objetivos educativos y los resultados reales del aprendizaje.

En cambio, este artículo está basado en una premisa difcrente sobre la enseñanza y el aprendizaje. Se supone que los individuos construyen su propio conocimiento; por tanto, el currículo es concebido, quizás más provechosamente, como el conjunto de experiencias que permiten que los estudiantes desarrollen las destrezas

\section{REFERENCLAS BIBLIOGRÁFICAS}

ANDERSSON, B. and KARRQUIST, C., 1983, How Swedish pupils, aged $12-15$ years, understand light and its properties. Eur. J. Sci. Educ. 5 (4), 387-402.

ATKINSON, P. y DELAMONT, S., 1976, Mock-ups and cock-ups: the stage management of guided discovery instruction. In : Hammersley and Woods, Process of Schooling. R.K.P.

BROOK, A. and DRIVER, R., 1986, The construction of meaning and conceptual change in classroom settings: $\mathrm{Ca}$ se studies in the learning of energy. Children's Learning in Science monograph. Centre for Studies in Science and Mathematics Education, University of Leeds.

CHAMPAGNE, A.B., KLOPFER, L.E. and GUNSTONE, R.F., 1982, Cognitive research and the design of science instruction. Educational Psychologist, 17, 31-53.

CLAXTON, G., 1986, Mini theories: A preliminary model for learning science Manuscript. Centre for Educational Studies, Kings's College London, Chelsea Campus, London.

CHILDREN'S LEARNING IN SCIENCE PROJECT (CLIS), CLIS in the Classroom Approaches to Teaching, Centre for Studies in Science and Mathematics Education, University of Leeds, Leeds. e ideas deseadas. Adoptar esta concepción tiene ciertas consecuencias:

a. Significa que los diseñadores del curriculo no sólo necesitan saber de la materia a enseñar, sino del modo en que se produce el aprendizaje. En las primeras secciones de este articulo se ha dado alguna indicación sobre la información que tenemos sobre el pensamiento y el aprendizaje de los estudiantes. Quedan, no obstante, muchas cuestiones abiertas.

b. Más que tomarse como un «a priori», el currículo como conjunto de actividades se constituye en sí mis mo en un objeto de investigación. Únicamente mediante control cuidadoso y retroalimentación a partir de materiales en uso seremos capaces de producir actividades de aprendizaje que estén adaptados a los modos de pensar de los estudiantes.

c. El desarrollo del profesor es un aspecto del de sarrollo del curriculo. Las experiencias que tienen los estudiantes en el aula están directamente influidas por el modo en que trabajan los profesores. Dar oportunidades a los estudiantes para que construyan por ellos mismos sentido de las experiencias de aprendizaje puede suponer cambios en la manera en que muchos profesores piensan y trabajan. Promover el cambio en la enseñanza puede necesitar una investigación análoga a la de promover el aprendizaje de los estudiantes.

DRIVER, R., 1983, The pupil as scientist? Open University Press, Milton Keynes.

DRIVER, R., GUESNE, E. and TIBERGHIEN, A. (eds.) Children's Ideas in Science, Open University Press.

DRIVER, R. and OLDHAM, V., 1986, A constructivist approach to curriculum development in science. Studies in Science Education, 13, 105-122.

DUIT, R., IUNG, W. and von RHONECK, C. (eds.), 1985, Aspects of understanding electricity, I.P.N., Kiel.

ENGEL CLOUGH, E. and DRIVER, R., 1985, What do children understand about pressure in fluids? Research in Science and Technology Education, 3 (2), 133-144.

ENGEL CLOUGH, E. and DRIVER, R., 1986, Consistency in the use of students' conceptual frameworks across different task contexts. Science Education, 70 (4), 473-496.

ERICKSON, G.L., 1979, Children's conceptions of heat and temperature. Science Education 63 (2), 221-230.

ERICKSON, G. and TIBERGHIEN, A., 1985, Heat and Temperature. In : Driver R., Guesne E. and Tiberghien A. (eds) Children's Ideas in Science, Open University Press. 
GAMBLE, R., 1986, Simple equations in physics. Eur. $J$. Sci. Educ. $8(1), 27-37$.

GUESNE, E., 1984, Children's Ideas about Light. New Trends in Physics Teaching Vol. IV. UNESCO.

GUNSTONE, R, and WHITE, R., 1981, Understanding of Gravity. Science Educ. 65 (3) 291-299.

HAWKINS, D., 1978, Critical Barriers in Science Learning. Outlook 29, 3-22.

HELM, H. and NOVAK, J., 1983, Proceedings of the International Seminar: Misconceptions in Science and Mat. hematics, Comeil University, Ithaca, U.S.A.

HEWSON, P. and HEWSON, M., 1984, The role of conceptual conflict in conceptual change and the design of science instruction, Instructional Science, 13, 1-13.

JUNG, W., PFUNDT, H., and RHONECK, C., 1982, Problems concerning students" representation of physics and chem istry knowledge Selbstuerlag, Pedagogische Hochschule, Ludwigsburg.

KOERTGE, N., 1970. Toward an integration of content and method in the science curriculum. Curriculum Theory Network, 4, 26-44.

LARKIN, J.H., 1983, The role of problem representation in physics. In: Gentner D. and Stevens A. (eds.) Mental Models L.E.A.

MILLER, S., ROBINSON, D. and DRIVER, R., 1985, Secondary students' ideas about air and air pressure. In: Bell B., Watts D.M. and Ellington K. (eds.) Learning, Doing and Understanding in Science S.S.C.R., London.

NUSSBAUM, J. and NOVICK, S., 1982, Alternative frameworks, conceptual conflict and accommodation: towards a principled teaching strategy. Instructional Science, $I$, 183-200.

OSBORNE, R. and FREYBERG, P. (eds.), 1985, Learning in Science. Heinemann.

REIF, F., 1986, Interpretation of scientific or mathematical concepts; cognitive issues and instructional implications. Report No CES-1, Department of Physics and School of Education, University of California, Berkeley, U.S.A.
ROWELL, J.A. and DAWSON, C.J., 1983, Laboratory counter examples and the growth of understanding in science. European Journal of Science Education 4 (3), 299-309.

SERE, M.G., 1982, A study of some frameworks used by pupils aged 11 to 13 years in the interpretation of air pressure. European Journal of Science Education 4 (3), 299-309.

SHIPSTONE, D., 1985, Electrictiy in simple circuits, In: Driver, R., Guesne E. and Tiberghien A. (eds.) Children's Ideas in Science, Open University Press.

SJOBERG, S. and LIE, S., 1981, Ideas about force and movement among Norwegian pupils and students. Report 81+11, Institute of Physics Report Series, University of Oslo, Norway.

SOLOMON, J., 1983, Learning about energy: how pupils think in two domains. European Journal of Science Edu. cation, 5 (1), 49-59.

STRAUSS, S. and STAVY, R., 1982, U-shaped behavioral growth: Implications for theories of development. Review of Child Development Research, Volume 6 (ed.) W. Hartup, University of Chicago Press.

VIENNOT, L., 1979, Spontaneous reasoning in elementary dynamics. European Journal of Science Education l (2), 202-222.

WELLINGTON, J.J., 1981, What's supposed to happen sir?: Some problems with discovery learning. School Science Review, 63, 163-173.

WENHAM, E.J. (Ed.), 1984, New trends in physics teaching Volume $I V$ UNESCO.

WEST, L. and PINES, A. (Ed.), 1985, Cognitive Structure and Conceptual Change. Academic Press.

WIGHTMAN, T., GREEN, P. and SCOTT, P., 1986, The construction of meaning and conceptual change in classroom settings: Case studies on the particulate theory of matter. Children's Learning in Science monograph, Centre for Studies in Science and Mathematics Education, Uni. versity of Leeds.

WITTROCK, M.C., 1974, Learning as a generative process. Educational Psychology, 11, 87-95. 